9-ORIGINAL ARTICLE

CLINICAL INVESTIGATION

\title{
Leptin and leptin receptor expressions in prostate tumors may predict disease aggressiveness? ${ }^{1}$
}

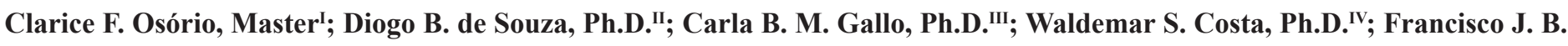 \\ Sampaio, M.D., Ph.D ${ }^{v}$ \\ DOI: http://dx.doi.org/10.1590/S0102-86502014001700009 \\ ${ }^{\text {I }}$ Researcher, Urogenital Research Unit, State University of Rio de Janeiro (UERJ), Rio de Janeiro-RJ, Brazil. Performed the experiment, \\ histomorphometrical examination, wrote the manuscript, read and approved the final manuscript. \\ II Associate Professor, Urogenital Research Unit, State University of Rio de Janeiro (UERJ), Rio de Janeiro-RJ, Brazil. Designed the study, supervised \\ all parts of the experiment, wrote the manuscript, read and approved the final manuscript.

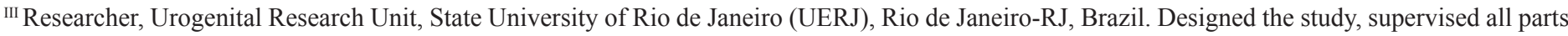 \\ of the experiment, wrote the manuscript, read and approved the final manuscript. \\ ${ }^{\text {IV }}$ Associate Professor, Urogenital Research Unit, State University of Rio de Janeiro (UERJ), Rio de Janeiro-RJ, Brazil. Designed the study, supervised \\ all parts of the experiment, wrote the manuscript, read and approved the final manuscript. \\ ${ }^{v}$ Full Professor, CNPq 1A researcher, Urogenital Research Unit, State University of Rio de Janeiro (UERJ), Rio de Janeiro-RJ, Brazil. Critical revisions, \\ statistical analysis, read and approved the final manuscript.
}

\section{ABSTRACT}

PURPOSE: The aim of this study was to evaluate the expression of leptin and its receptor in histological sections of prostate tumors, and their association with prognostic factors.

METHODS: A total of 532 surgical specimens from prostate cancer were studied. After histopathological diagnosis, the samples were included in tissue microarrays containing cores from tumor and non-tumor (benign prostatic hyperplasia) areas. These were immunostained with anti-leptin and anti-leptin-receptor antibodies. Objective and subjective analyses were performed. Student's-t-test and ANOVA were used to compare mean values, and linear regression was used to evaluate the correlation between histological results and prognostic indicators.

RESULTS: Leptin receptor expression was reduced in tumors with a positive surgical margin, urethral margin involvement, and seminal vesicles invasion. Further, there was a negative correlation between the expression of leptin receptor in tumor areas and the sum of prognostic factors, suggesting that leptin receptor may predict the aggressiveness of disease.

CONCLUSION: Our findings suggest that leptin receptor expression is a potential prognostic factor for PCa. Further investigation is needed to support the use of leptin receptor as a novel biomarker, although leptin itself does not seem to predict the aggressiveness of prostate cancer.

Key words: prostate cancer, leptin, leptin receptor 


\section{Introduction}

Leptin is a hormone responsible for regulating body fat. It is secreted by adipocytes, and its serum level varies with body weight and physical activity. Like other hormones, leptin needs to bind a receptor to exert its effects ${ }^{1,2}$. Therefore, not only the amount of leptin but also the number of leptin receptors on a target cell is important in determining its activity.

In vitro studies have shown that leptin is a mitogenic factor in several malignancies, including endothelial, breast, colon, prostate, and esophageal cancers ${ }^{3,4}$. Leptin also stimulates the growth and migration of neoplastic cells in vitro and increases the proliferation of prostate cancer ( $\mathrm{PCa}$ ) cells via the suppression of apoptosis, thus mediating more aggressive biological behavior ${ }^{3,4}$.

Leptin only stimulates cell proliferation in some androgen-independent PCa-derived cell lines, but not in cells derived from androgen-dependent tumors, despite both cell types expressing functional leptin receptor isoforms ${ }^{5}$. Leptin has been shown to have a role in the development of PCa via testosterone and obesity-related factors and influences the cellular differentiation and progression of this malignancy ${ }^{6,7}$.

Analysis of leptin expression in $\mathrm{PCa}$ and benign prostatic hyperplasia (BPH) has shown that it is more abundant in the former. Leptin expression is greater still in metastatic and locally advanced prostate tumors compared to localized tumors. Therefore, it has been suggested that the increased expression of leptin is related to the progression and degree of malignancy of this disease ${ }^{8,9}$.

Different methods have been used to determine the prognosis of PCa. These include histopathological classification through the scoring system devised by Gleason, which is widely used, as it correlates with disease progression ${ }^{10,11}$. The Gleason's score correlates with the levels of prostate specific antigen (PSA), clinical and pathological staging of the disease, frequency of corpuscle apoptosis, over expression of p53, the incidence of lymph node and bone metastases and response to therapy ${ }^{12,13}$. Although the Gleason score is the best method for determining a prognosis in $\mathrm{PCa}$, differences in scores may occur when different pathologists analyze the same sample.

In addition to Gleason score, the prognosis of $\mathrm{PCa}$ is also related to a number of other factors, including pathological staging criteria such as capsular penetration, positive surgical margin, the involvement of urethral and vesical margins, seminal vesicle invasion, and lymph node involvement ${ }^{13-15}$.

This study was designed to evaluate, by quantitative computational methods, the expression of leptin and leptin receptor in $\mathrm{PCa}$ and to study its association with the presence of positive surgical margins, vesical margin involvement, seminal vesicle invasion, bilateral involvement of the lobes, perineural invasion, and vascular embolization.

\section{Methods}

A total of 532 cases of prostatic adenocarcinoma diagnosed using surgical samples from open radical prostatectomy were evaluated. These samples were obtained from a tissue bank maintained by the "Laboratório HPCF Anatomia Patológica e Citopatologia Ltda.”. The procedures were in accord with the Ethics Committee of Pedro Ernesto University Hospital (State University of Rio de Janeiro), and was formally approved by this comitee.

The samples were fixed in formaldehyde for 8 to $12 \mathrm{~h}$, and the surgical margins were labeled with India ink. Fragments of the vesicle (higher) and urethral (lower) limits were removed for histological examination. The tissue sections were stained with hematoxylin and eosin and visualized by light microscopy (Nikon YS100, Tokyo, Japan). The diagnosis was confirmed to be prostatic adenocarcinoma in each case, and the samples were classified by Gleason score ${ }^{10}$. For each section, areas of tumor and BPH were identified.

We analyzed the following parameters: perineural invasion, vascular embolization, bilateral involvement of the lobes, seminal vesicle invasion, vesical margin involvement, urethral margin involvement, and surgical resection margin. Each of these parameters was classified as 0 (absent) or 1 (present). The sum of the results for these different factors was analyzed. A score of 0 was given when none of these features were present, and a score of 7 was given when they were all present.

Samples embedded in paraffin (donor block) were selected and labeled in blue to mark tumor fields and red to mark BPH fields.

From each selected area of the donor block, a core was collected by direct puncture of the marked area with 1-mm diameter needle. These cores were included in a new block (receiver block). Four blocks of tissue microarray (TMA) with a total of 1,064 specimens (tumor and BPH532, 532) were prepared. Histological sections were cut from these four TMAs.

The expressions of leptin and its receptor were assayed using the Avidin Biotin method with specific antibodies: polyclonal anti-leptin (Leptin L3410, Sigma) and the polyclonal anti-receptor of leptin (Anti-Ob-R SC-1834, Santa Cruz).For each primary antibody, simultaneous negative controls were used, in which the primary antibody was replaced with PBS, as well as positive controls, for which placental tissue was used. The sections were labeled with a biotinylated secondary antibody (Biocare; code 
M4BD534L, California, USA) and stained with diaminobenzidine tetrahydrochloride (Biocare; code M4BD534L, California, USA).

For quantitative analysis, tumor and non-tumor fields were selected from the TMA and photographed at a magnification of $400 \times$ with an Olympus DP70 digital camera coupled to an Olympus BX51microscope (Olympus, Tokyo, Japan). The images were captured and analyzed using ImageJ software (version $1.45 \mathrm{~s}$, National Institutes of Health, Bethesda, USA) and a grid cell counter.

For the semi-quantitative analysis, two pathologists evaluated the immunolabeled sections subjectively from $\times 400$ magnification images. The staining was interpreted and classified in accordance with the following scores: negative (0), weakly positive (1), positive (2), and strongly positive (3). The result for each section was the average score of the two observers.

A statistical analysis was performed using Student's t-test and an analysis of variance (ANOVA) in order to compare the quantitative results among specimens with low $(\leq 6)$, intermediate

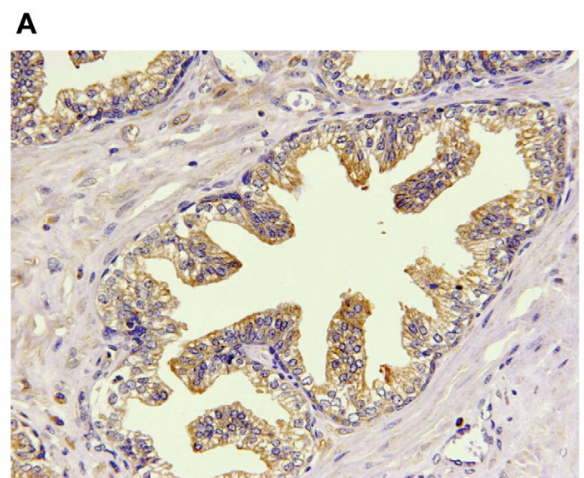

(7), and high $(\geq 8)$ Gleason scores. Linear regressions were performed between the quantitative results and the different prognostic factors that were studied in order to identify associations between leptin and leptin receptor staining and the various prognostic factors. All values were given as the mean \pm standard deviation.

\section{Results}

The analyzed samples were from patients with a mean age of $63 \pm 8$ years (range, 40-86 years), with an average serum PSA level of $7.2 \pm 4.7 \mathrm{ng} / \mathrm{ml}$. In the quantitative analyses, $59.7 \%$ $\pm 17.4 \%$ of the tumor cores and $58.6 \% \pm 20.2 \%$ of the non-tumor cores were positive for leptin. With respect to leptin receptor expression, $74.4 \% \pm 17.1 \%$ of the tumor cores and $75.4 \% \pm 17.1 \%$ of the non-tumor cores were positive. No significant difference was found between the expression of leptin and its receptor in the tumor and non-tumor cores (Figure 1 and Figure 2).

\section{B}

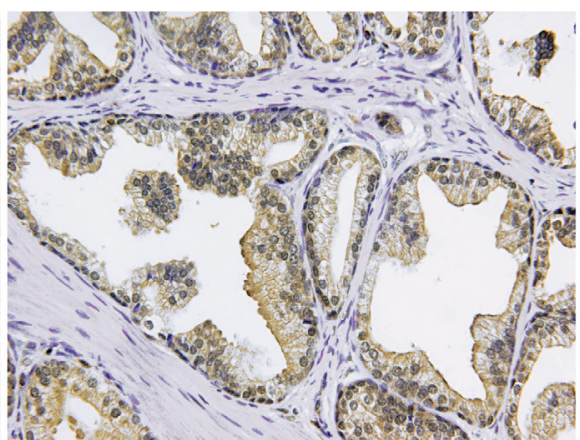

Figure 1. Photomicrographs of non-tumor prostate cores immunolabeled with anti-leptin (a) and anti-leptin receptor (b) antibodies (400×)

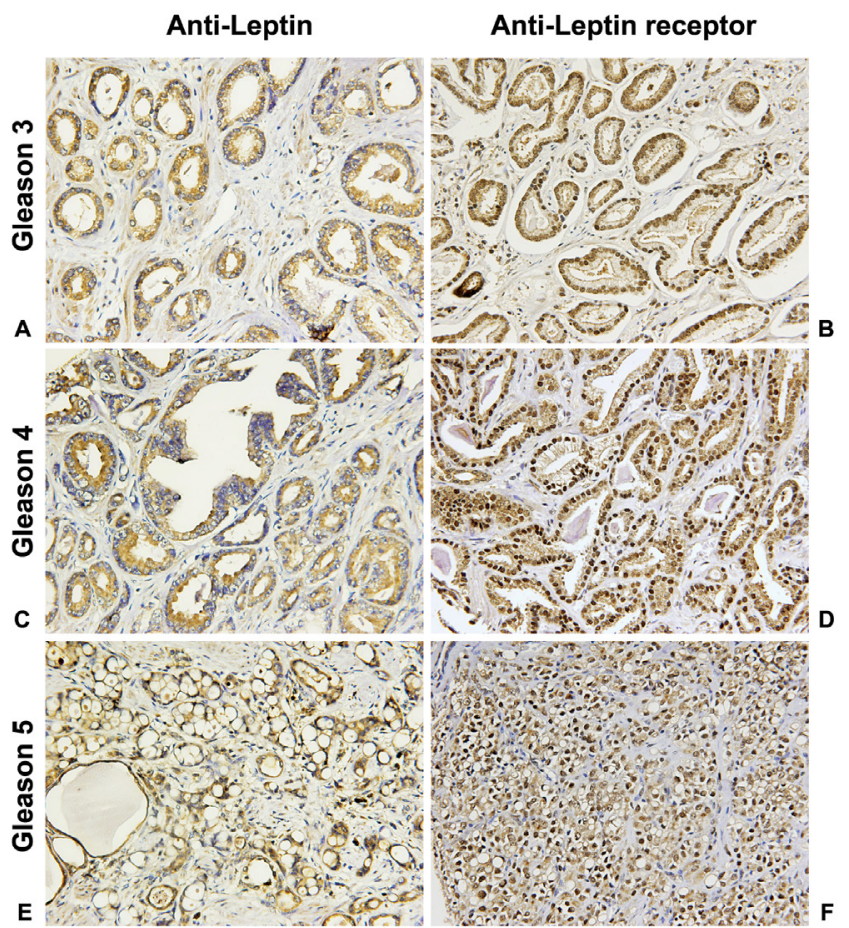

Figure 2. Photomicrographs of prostate tumor cores. Sections with different Gleason grades immunolabeled with anti-leptin antibodies (a, c, e) and anti-leptin receptor antibodies $(b, d, f) .(400 \times)$ 
Additionally, no significant difference was found between the expression of leptin in tumors with different Gleason scores: low $(\leq 6)$, intermediate $(7)$, and high $(\geq 8)$.

There was also no significant difference in the expression of leptin with respect to the different prognostic factors analyzed. However, the expression of leptin receptor was reduced by $11.3 \%, 4.6 \%$, and $6.4 \%$ in tumors that exhibited urethral margin involvement, a positive surgical margin, and seminal vesicle invasion, respectively, in comparison to those that do not exhibited these factors (Figure 3).
A

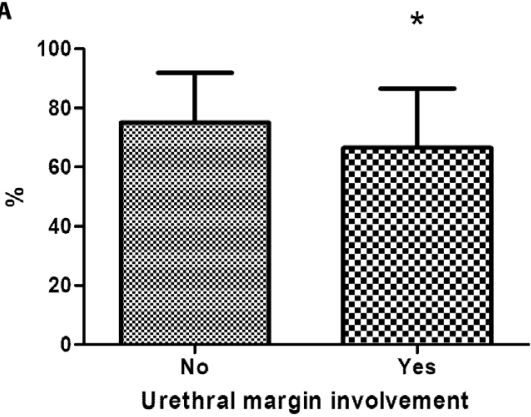

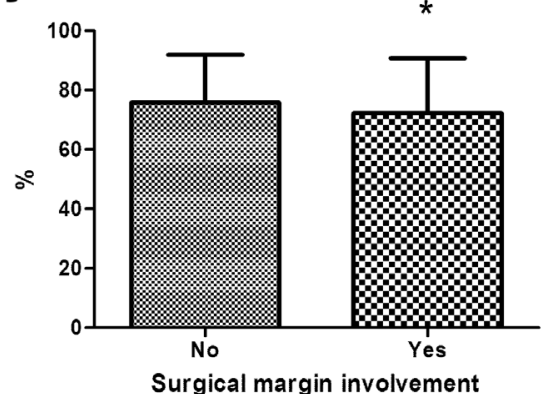

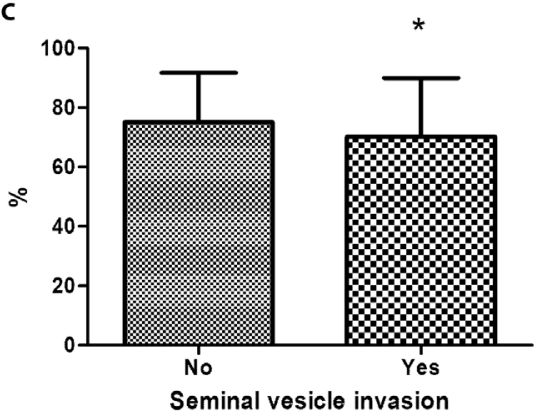

Figure 3. Proportion of prostate tumor cores expressing anti-leptin receptors. Receptors in samples (a) with or without urethral margin involvement, (b) with positive or negative surgical margins, and (c) with or without seminal vesicle invasion $(* \mathrm{p}<0.05)$

There was no significant difference in the expression of the leptin receptor with respect to the other prognostic factors.

There was no association between the tumor area positive for leptin expression and the Gleason score $\left(\mathrm{p}<0.05 ; \mathrm{r}^{2}=0.002\right)$ or the sum of the prognostic factors $\left(p<0.05 ; r^{2}=0.001\right)$. However, there was a strong correlation between the tumor area positive for leptin receptor expression and the sum of the prognostic factors analyzed $\left(\mathrm{p}=0.01 ; \mathrm{r}^{2}=0.025\right)$ (Figure 4$)$.

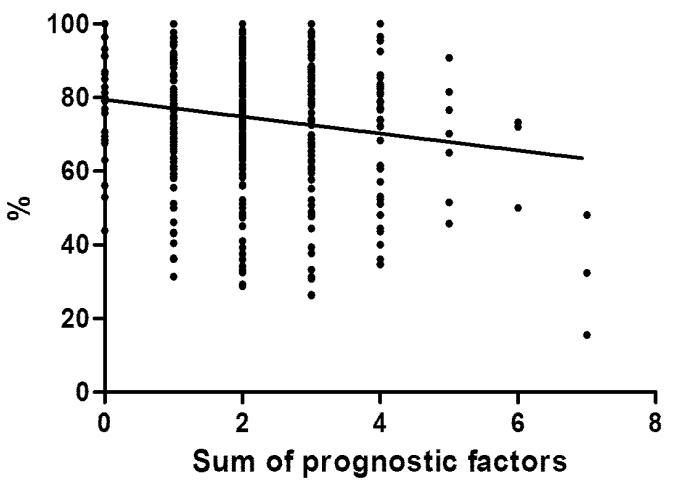

Figure 4. Correlation between the proportion of tumor area expressing leptin receptor and the sum of prognostic factors $\left(\mathrm{p}=0.001 ; \mathrm{r}^{2}=0.025\right)$.

In the semi-quantitative analyses, the mean score for expression of leptin was $0.44 \pm 0.52$ in the tumor and $0.11 \pm 0.23$ in the non-tumor area, whilst the mean expression of the leptin receptor was $1.4 \pm 0.5$ in the tumor and $1.1 \pm 0.4$ in the non-tumor area. However, there were significant differences in the staining scores recorded by the two observers. For leptin expression in the tumor, the difference between the averages of the observers was
$0.14 \pm 0.03(p<0.05)$, and for the non-tumor area, it was $0.05 \pm$ $0.01(\mathrm{p}<0.05)$. For the analysis of leptin receptor expression in the tumor, the difference between the averages of the observers was $0.37 \pm 0.03(p<0.05)$ and for the non-tumor area it was $0.27 \pm 0.02$ $(\mathrm{p}<0.05)$.

\section{Discussion}

Patients with prostate cancer have elevated serum levels of leptin ${ }^{16}$, and this is correlated with tumor volume, histological classification, biochemical recurrence, metastasis, and disease progression, as well as an increase in mortality ${ }^{6}$. There is also a strong correlation between serum leptin levels and PSA 16, 17 . With respect to prostate tissue, leptin is expressed more strongly in tumors than in hyperplasic tissues; the expression of leptin was also elevated in locally advanced and metastatic tumors compared with localized tumors ${ }^{8}$. Therefore, increased leptin expression was predicted to be associated with progression and malignancy of disease.

However, we found no difference between the expression of leptin or its receptor between tumor and non-tumor tissue. This may relate to the methodology used for the quantification of leptin. In our study, we performed a computational analysis rather than an analysis based on a subjective method that depends on the observer's interpretation of immunolabeling intensity as being either strong or weak ${ }^{8}$. Our results confirm this assertion, with a significant difference between the subjective analysis of the two observers quantifying leptin and its receptor. Subjective methods for the analysis of morphological changes are highly influenced 
by the observer and thus, often difficult to reproduce. Quantitative computational methods should be used instead of subjective methods, as the latter may show a greater variation ${ }^{18}$.

We also reviewed pathological data, such as perineural and seminal vesicles invasion, vascular embolization, bilateral involvement of the lobes, vesical and urethral margin involvement, and positive surgical margin. In addition to the Gleason score, each of these factors influence prognosis to some extent ${ }^{19}$. It has been suggested that leptin expression is also a potential prognostic factor in PCa, although we found no association between leptin expression in tumors and any of these other prognostic factors.

However, we found that the expression of leptin receptor was significantly lower in tumors exhibiting a number of prognostic factors, including urethral margin involvement, surgical margin involvement, and seminal vesicles involvement. There was also a negative correlation between the sum of these prognostic factors and the expression of leptin receptor in prostate tumors, suggesting that lower expression of leptin receptor may indicate a more aggressive tumor. Therefore, leptin may influence the aggressiveness of prostate cancer indirectly by changing the availability of its receptor.

It was not possible to classify tumors as localized, locally advanced, or metastatic ${ }^{20}$ because we had no clinical data to rule out or confirm the presence of metastasis. Despite the large number of samples and the numerous parameters studied in this work, a study that considers more clinical data and that which involves a longer follow-up period is needed to corroborate our findings.

\section{Conclusions}

Leptin receptor is a possible prognostic factor in $\mathrm{PCa}$, as its expression is lower in the presence of a surgical margin involvement, urethral margin involvement, and seminal vesicle invasion, and that there is a negative correlation between the sum of key prognostic factors and leptin receptor expression.. However, we found that the expression of leptin itself on tumor histological sections had no apparent prognostic significance.

\section{References}

1. Frühbeck G. Intracellular signalling pathways activated by leptin. Biochem J. 2006; 393: 7-20.

2. Ray A. Adipokine leptin in obesity-related pathology of breast cancer. J Biosc 2012; 37: 289-94.

3. Somasundar P, Frankenberry KA, Skinnner H, Vedula G, Mcfadden DW, Riggs D, Jackson B, Vangilder R,Hileman SM, Vona-Davis LC. Prostate cancer cell proliferation is influenced by leptin. J Surg Res. 2004; 118: 71-82.

4. Lang K, Ratke J. Leptin and adiponectin: new players in the field or tumor cell and leukocyte migration. Cell Commun Signal. 2009; 7: $1-10$.

5. Onuma M, Bud JD, Rummel TL, Iwamoto Y. Prostate cancer celladipocyte interaction. J Biol Chem. 2003; 278: 42660-7.

6. Saglam K, Aydur E, Yilmaz M, Göktas S. Leptin influences cellular differentiation and progression in prostate cancer. J Urol. 2003; 169: 1308-11.

7. Chang S, Hursting SD, Contois JH, Strom SS, Yamamura Y, Yamamura Y, Babaian RJ, Troncoso P, Scardino PS, Wheeler TM, Amos CI, Spitz MR. Leptin and prostate cancer. Prostate. 2001; 46: 62-7

8. Kim JH, Lee SY, Myung SC, Kim YS, Kim T-H, Kim MK. Clinical significance of leptin and leptin receptor expressions in prostate tissues. Asian J Androl. 2008; 10: 923-8.

9. Tewari R, Rajender S, Natu SM, Goel A, Dalela D, Goel MM, Tondon P. Significance of obesity markers and adipocytokines in high grade and high stage prostate cancer in North Indian men - a cross-sectional study. Cytokine. 2013; 63: 130-4.

10. Hack EE, Siemens DR, Groome PA. The relationship between adiposity and Gleason score in men with localized prostate cancer. Prostate. 2010; 70: 1683-91.

11. Gleason DF. Classification of prostatic carcinomas. Cancer Chemother Rep. 1966; 50: 125-8.

12. Partin AW, Yoo J, Carter HB, Pearson JD, Chan DW, Epstein JI, Walsh PC. The use of prostate specific antigen, clinical stage and Gleason score to predict pathological stage in men with localized prostate cancer. J Urol. 1993; 150: 110-4.

13. Gleason DF, Mellinger GT. Prediction of prognosis for prostatic adenocarcinoma by combined histological grading and clinical staging. J Urol. 1974; 111: 58-64.

14. Kryvenko ON, Gupta NS, Virani N, Schultz D, Gomez J, Amin A, Lane Z, Epstein JI Gleason score 7 adenocarcinoma of the prostate with lymph node metastases: analysis of 184 radical prostatectomy specimens. Arch Pathol Lab Med. 2013; 137: 610-7.

15. Yossepowitch O, Briganti A, Eastham JA, Epstein J, Graefen M, Montironi R, Touijer K. Positive Surgical Margins After Radical Prostatectomy: A Systematic Review and Contemporary Update. Eur Urol. 2014; 65: 303-13.

16. Drew JE. Molecular mechanisms linking adipokines to obesity-related colon cancer: focus on leptin. Proc Nutr Soc. 2012; 71: 175-80.

17. Singh SK, Grifson JJ, Mavuduru RS, Agarwal MM, Mandal AK, Jha V. Serum leptin: a marker of prostate cancer irrespective of obesity. Cancer Biomark. 2010; 7: 11-5.

18. Mandarim-de-Lacerda CA. Stereological tools in biomedical research. An Acad Braz Cienc. 2003; 75: 469-86.

19. Bostwick DG, Qian J, Bergstralh E, Dundore P, Dugan J, Myers RP, Oesterling JE. Prediction of capsular perforation and seminal vesicle invasion in prostate cancer. J Urol. 1996; 155:1361-7.

20. Rosai J, Ackerman LV. Rosai \& Ackerman's Surgical Pathology. Philadelphia: W. B. Saunders. 2004, 2894 p.

\section{Correspondence}

Prof. Francisco J. B. Sampaio, M.D., Ph.D.

Urogenital Research Unit

Blvd. 28 de Setembro, 87 - Fundos, Brazil

Zip. 20551-030

Phone/Fax number: +55 21 2868-8021

sampaio@urogenitalresearch.org

${ }^{1}$ The work was performed at the Urogenital Research Unit, State University of Rio de Janeiro. 